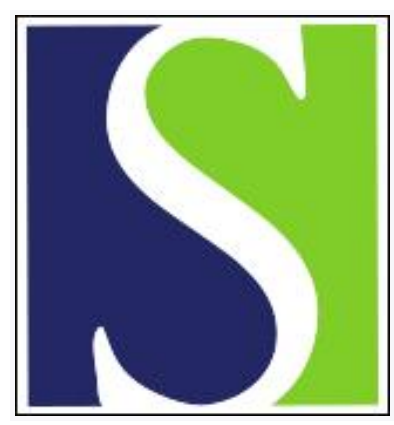

Scand J Work Environ Health 1998;24(3):183-189

https://doi.org/10.5271/sjweh.297

Issue date: Jun 1998

Nocturnal excretion of a urinary melatonin metabolite among electric utility workers

by Burch JB, Reif JS, Yost MG, Keefe TJ, Pitrat CA

The following article refers to this text: $2004 ; 30$ suppl 1:1-80

Key terms: 6-hydroxymelatonin sulfate; electromagnetic fields; human; magnetic fields; pineal

This article in PubMed: www.ncbi.nlm.nih.gov/pubmed/9710370

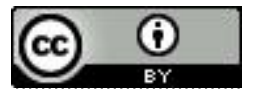




\title{
Nocturnal excretion of a urinary melatonin metabolite among electric utility workers
}

\author{
by James B Burch, PhD, ${ }^{1}$ John S Reif, DVM, Michael G Yost, PhD, ${ }^{2}$ Thomas J Keefe, PhD, ${ }^{1}$ Charles A \\ Pitrat, MS
}

Burch JB, Reif JS, Yost MG, Keefe TJ, Pitrat CA. Nocturnal excretion of a urinary melatonin metabolite among electric utility workers. Scand J Work Environ Health 1998:24(3):183-189.

\begin{abstract}
Objectives The effects of $60-\mathrm{Hz}$ magnetic field and ambient light exposures on the pineal hormone melatonin were studied among electric utility workers.

Methods Personal exposure was measured at 15-second intervals over 3 consecutive 24-hour periods. Exposure metrics based on magnetic field intensity, intermittence, or temporal stability were calculated for periods of work, home, and sleep. A rate-of-change metric (RCM) was used to estimate intermittence, and the standardized RCM (RCMS $=\mathrm{RCM} /$ standard deviation) was used to evaluate temporal stability. The effects of magnetic field exposure on total overnight 6-hydroxymelatonin sulfate (6-OHMS) excretion and creatinine-adjusted nocturnal 6-OHMS (6OHMS/cr) concentration were analyzed with adjustment for age, month, and light exposure.

Results Magnetic field intensity, intermittence, or cumulative exposure had little influence on nocturnal 6-OHMS excretion. Residential RCMS magnetic field exposures were associated with lower nocturnal 6-OHMS/cr concentrations. In multivariate statistical analyses, the interaction term for geometric mean and RCMS magnetic field exposures at home was associated with lower nocturnal 6-OHMS/cr and overnight 6-OHMS levels. Modest reductions in the mean 6-OHMS levels occurred after RCMS exposures during work. The greatest reductions occurred when RCMS exposures both at work and at home were combined; therefore the effects of temporally stable magnetic fields may be integrated over a large portion of the day.

Conclusions Results from this study provide evidence that temporally stable magnetic field exposures are associated with reduced nocturnal 6-OHMS excretion in humans.
\end{abstract}

Key terms electromagnetic fields, human, 6-hydroxymelatonin sulfate, $60 \mathrm{~Hz}$, magnetic fields, pineal.

The potential health effects associated with exposure to power frequency $(50 / 60 \mathrm{~Hz})$ magnetic fields have received considerable attention in recent years, due in part to the pervasiveness of such exposures in the home and workplace. The characterization of human biological responses to magnetic field exposures is critical in determining whether such exposures result in adverse health effects. Some magnetic field effects may be mediated through reduced secretion of the hormone melatonin. Melatonin secretion follows a diurnal rhythm (night high, day low) that is synchronized by ambient light exposure (1). Through this mechanism, melatonin influences sleep and other physiological processes with circadian rhythms $(2,3)$. Melatonin is also associated with suppressed tumor growth (3-6), enhanced immunity (6-9), antioxidant effects (10-12), and reduced secretion of tumor-promoting hormones $(13,14)$. Decreased melatonin production could therefore have important biological consequences.
Although a reduction in melatonin synthesis following exposure to magnetic fields has been reported for a variety of experimental animal models (15), only a few studies have attempted to determine whether such effects occur in humans. In a study of electric blanket users, Wilson et al (16) found a reduction in nocturnal urinary concentrations of the major melatonin metabolite 6-hydroxymelatonin sulfate (6-OHMS or 6-sulfatoxymelatonin) in some persons after 8 weeks of electric blanket use. Cessation of electric blanket use was accompanied by an increase in 6-OHMS excretion (16). A reduction in early evening, but not overnight, 6-OHMS excretion was reported recently in a study of railway workers with occupational exposure to $16.7 \mathrm{~Hz}$ magnetic fields (17).

Elevated magnetic field exposures have been reported for electric utility workers (18-21). Numerous epidemiologic studies have identified this occupational group as having an elevated risk for developing leukemia (22)

1 Department of Environmental Health, Colorado State University, Fort Collins, Colorado, United States.

2 Department of Environmental Health, University of Washington, Seattle, Washington, United States.

Reprint requests to: Dr James Burch, Department of Environmental Health, Colorado State University, Fort Collins, CO 80523, United States. 
or brain cancer (23). Therefore, this study was designed to test the hypothesis that electric utility workers exposed to magnetic fields exhibit a decrease in nocturnal melatonin biosynthesis.

\section{Subjects and methods}

The study population comprised 142 male electric power utility workers aged 20 to 60 years. Generation workers [N=29] (utility electricians and operators), distribution workers [ $\mathrm{N}=56]$ (linemen and substation operators), and a comparison group of utility maintenance and administrative staff $[\mathrm{N}=57]$ were studied concurrently over a 1year period. The mean age was 41 (SD 0.6) years; approximately $90 \%$ were Caucasian and non-Hispanic. All the subjects worked a normal daytime shift during their participation in the study. A questionnaire was administered to collect information concerning personal (age, race, body mass index), occupational (job title, employment duration, use of cell phones and other equipment, physical activity, work with chemicals), life-style (tobacco and alcohol use, sleep habits, electrical appliance use, exercise), and medical factors (medication, disease history) that might influence magnetic field exposure or melatonin production. None of the subjects were taking exogenous melatonin.

\section{Exposure assessment}

Personal exposure to magnetic fields and ambient light was measured over a period of 3 consecutive workdays, and during the night preceding the first day of work. Twenty-four hour magnetic field and light exposures were recorded at 15-second intervals with EMDEX $\mathrm{C}$ meters (19). Light exposure was measured by a Grasby Optronics photometric sensor adapted to the external jack of the EMDEX. The meter was worn in a belt pack with the subject at work and off duty; it was placed beside the bed adjacent to the waist during the worker's sleep. Calibration logs and recordings of magnetic fields, light, and motion were inspected, and data were excluded if the meter was out of calibration, malfunctioning, or not worn. The participants logged their times at work, at home, and in bed, and exposures were partitioned accordingly. Home exposures were comprised mainly of time spent at the residence in the evening with a small component due to time at home prior to work.

\section{Exposure metrics}

Magnetic field and light exposure metrics were calculated for each exposure period and day of study. The arithmetic time-weighted average (TWA) was used to summarize personal light exposure. Magnetic field exposure metrics were selected a priori and included the arithmetic
TWA, the geometric TWA, cumulative exposure, and cumulative exposure above $0.2 \mu \mathrm{T}(24,25)$. Two other metrics were calculated according to proposed mechanisms of magnetic field action. Exposure to fields with many switching events may have important biological implications (26-28). Therefore, a "rate of change metric" (RCM) based on the root-mean-square variation in successive magnetic field measurements was used to measure the intermittence of exposure (29):

$$
\operatorname{RCM}(\mu \mathrm{T} / 15 \mathrm{~s})=\sqrt{ }\left[\sum\left(\mathrm{MF}_{1}-\mathrm{MF}_{2}\right)^{2} /(\mathrm{n}-1)\right]
$$

where $\mathrm{MF}_{1}$ and $\mathrm{MF}_{2}$ are successive 15-second magnetic field measurements and $n$ is the number of measurements within a given exposure period. The RCM provides an estimate of both the variability and the first-lag autocorrelation in a series of measurements. Higher RCM values indicate greater variability or less autocorrelation between successive readings or both. Others have suggested that temporally stable magnetic fields induce biological effects $(30-32)$. The standardized RCM (RCMS) was therefore derived as follows:

RCMS (per $15 \mathrm{~s})=\mathrm{RCM} / \mathrm{SD}$,

where SD is the standard deviation of the magnetic field measurements in a given period. The RCMS estimates the first-lag autocorrelation. Low RCMS values correspond to relatively small differences between successive measurements and represent magnetic field exposures that are stable over time. Thus low RCMS values should be directly associated with low 6-OHMS levels.

The geometric mean and RCMS magnetic field exposures are summarized in table 1 by worker group and exposure period. In general, the measures of magnetic field intensity correlated well. The geometric mean magnetic field exposures at work were higher for the generation workers than for the comparison workers $(\mathrm{P}<0.01)$. For comparison with other studies, the arithmetic means for the workplace exposures were $0.23,0.32$, and $0.15 \mu \mathrm{T}$ for the distribution, generation, and comparison workers, respectively.

\section{Determination of 6-hydroxymelatonin sulfate}

Morning urine samples were collected daily for 4 days to determine the 6-OHMS levels. The base-line sample was obtained prior to the beginning of the workweek. The participants then submitted a morning sample on each of 3 consecutive workdays. Night-time and first morning voids were pooled to provide a total overnight sample. Melatonin production was assessed by a radioimmunoassay of urinary 6-OHMS concentrations $(33,34)$ (CIDtech, Mississauga, Ontario, Canada), which follow a diurnal pattern that is highly correlated with circulating melatonin (35). Total overnight 6-OHMS excretion and the nocturnal 6-OHMS concentration adjusted for creatinine (6-OHMS/cr) were calculated for each day. 


\section{Data analyses}

Statistical analyses were performed using log-transformed data ( $\log$ of the reciprocal for RCMS). Magnetic field exposures were compared among the distribution, generation, and comparison groups with a repeated-measures analysis of variance. Analyses for magnetic field effects were adjusted for age, month of participation, and TWA light exposure for the same period using Proc Mixed for repeated measures (SAS Institute Inc, Cary, NC, USA). Additional analyses were performed to evaluate potential confounding by the questionnaire variables; the results were essentially unchanged from those presented in this text. The potential effects of magnetic fields on the 6OHMS excretion were modeled in 2 ways. First, 6-OHMS excretion was analyzed using each magnetic field metric as a continuous variable with age, month, and light exposure included as covariates in the Proc Mixed analysis, along with "day" and "magnetic fields by day". Second, magnetic field exposures were divided into quartiles, and the least-squares means (adjusted for age, month, and light exposure) were estimated for the 6-OHMS for each quartile of exposure. The means in the lowest and highest exposure quartiles were compared by Fisher's least significant difference method.

\section{Results}

The overall mean of the overnight 6-OHMS excretion was 22.7 (SD 1.3) $\mu \mathrm{g}$, a value consistent with previously published data $(35,36)$. There was a statistically significant association between month of participation and both measures of 6-OHMS excretion $(\mathrm{P}<0.01)$; mean levels were higher in winter and lower in summer. Light exposures (TWA or cumulative lux) at home and during commutes from work to home were associated with lower 6-OHMS levels.

When each magnetic field metric was analyzed separately as a continuous variable, no statistically significant associations between 6-OHMS excretion and magnetic field intensity (TWA and cumulative exposures) or intermittence (RCM) were found. When RCMS was analyzed as a continuous variable, low RCMS exposures at home were associated with lower nocturnal 6-OHMS/cr concentrations $(\mathrm{P}<0.01)$ and lower overnight 6-OHMS excretion $(P=0.06)$. No statistically significant reductions in 6-OHMS were found for RCMS exposures during work or sleep.

Further analyses were performed to determine whether temporally stable magnetic field exposures that occurred at higher field strengths had more of an effect than stable exposures at lower intensities. The geometric mean, RCMS, and their interaction term were included in the analysis as continuous variables. The interaction term for the geometric mean and RCMS magnetic field exposure at home was associated with lower 6-OHMS/cr concentrations $(P<0.01)$ and with reduced overnight 6-OHMS excretion $(\mathrm{P}<0.01)$. Subjects with exposure to high-intensity magnetic fields that were also temporally stable had the lowest 6-OHMS levels. Similar results were obtained using the interaction term for RCMS with other intensity metrics at home. Interaction terms for RCMS with other exposure metrics during work or sleep were not associated with lower 6-OHMS levels.

The mean nocturnal 6-OHMS/cr concentrations and total overnight 6-OHMS excretion are presented by the quartile of the geometric mean and RCMS magnetic field exposures in tables 2 and 3, respectively. Quartile 4 represents the highest level of magnetic field intensity or temporal stability. Thus the mean 6-OHMS levels are arranged by increasing quartile of the geometric mean and decreasing quartile of the RCMS magnetic field exposure.

There was little evidence for reduced 6-OHMS excretion with increasing intensity of magnetic field exposure as measured by the geometric mean (table 2). However, the mean nocturnal 6-OHMS/cr concentrations were consistently lowest in subjects that were in the lowest quartile of RCMS exposure during work, home, or sleep (table 3). The difference between the highest and lowest quartiles was statistically significant for the home RCMS exposures $(\mathrm{P}<0.01)$. The mean overnight 6-OHMS excretion was also the lowest for the subjects that were in the lowest quartile of RCMS exposure although the differences

Table 1. Summary statistics for the magnetic field exposures of the male electric utility workers by exposure period. (RCMS = standardized rate of change metric)

\begin{tabular}{|c|c|c|c|c|c|c|c|c|c|c|c|c|}
\hline \multirow[t]{3}{*}{ Worker group } & \multicolumn{6}{|c|}{ Geometric mean $(\mu \mathrm{T})$} & \multicolumn{6}{|c|}{ RCMS (per 15s) } \\
\hline & \multicolumn{2}{|c|}{ Work ${ }^{a}$} & \multicolumn{2}{|c|}{ Homea } & \multicolumn{2}{|c|}{ Sleep $^{\mathrm{a}}$} & \multicolumn{2}{|c|}{ Work $^{\mathrm{a}}$} & \multicolumn{2}{|c|}{ Home $^{a}$} & \multicolumn{2}{|c|}{ Sleep } \\
\hline & Mean & SE & Mean & $\mathrm{SE}$ & Mean & SE & Mean & SE & Mean & SE & Mean & SE \\
\hline Distribution $(\mathrm{N}=56)$ & 0.10 & 0.03 & 0.11 & 0.04 & 0.08 & 0.05 & 0.64 & 0.04 & $0.55^{* *}$ & 0.03 & 0.50 & 0.04 \\
\hline Generation $(N=29)$ & $0.22^{\text {** }}$ & 0.07 & 0.14 & 0.05 & $0.11^{*}$ & 0.07 & 0.69 & 0.05 & 0.59 & 0.05 & $0.45^{*}$ & 0.04 \\
\hline Comparison $(\mathrm{N}=57)$ & 0.10 & 0.03 & 0.09 & 0.04 & 0.06 & 0.05 & 0.73 & 0.03 & 0.68 & 0.04 & 0.58 & 0.04 \\
\hline
\end{tabular}

a Mean and standard error of each exposure metric for days 1,2 , and 3 combined.

${ }^{*} P \leq 0.05$ versus comparison group, ${ }^{* *} P \leq 0.01$ versus comparison group. 
Table 2. Nocturnal 6-hydroxymelatonin sulfate (6-OHMS) excretion by the quartile of the geometric mean magnetic field exposure. (cr = creatinine)

\begin{tabular}{|c|c|c|c|c|c|c|c|c|}
\hline \multirow[t]{2}{*}{ Quartile of magnetic field exposure } & \multicolumn{4}{|c|}{ Nocturnal 6-OHMS / cr concentrationa (ng/mg) } & \multicolumn{4}{|c|}{ Total overnight 6-OHMS excretiona $(\mu \mathrm{g})$} \\
\hline & 1 & 2 & 3 & 4 & 1 & 2 & 3 & 4 \\
\hline Work & 29.6 & 25.2 & 30.0 & 28.5 & 17.7 & 15.0 & 17.8 & 14.8 \\
\hline Home & 27.4 & 27.3 & 31.3 & 25.9 & 16.0 & 16.9 & 16.6 & 15.6 \\
\hline Sleep & 25.3 & 31.9 & 30.6 & 26.8 & 15.1 & 17.7 & 17.7 & 16.3 \\
\hline
\end{tabular}

${ }^{a}$ Least-square means based on adjustment for age, month of participation, and mean light exposure during the same period.

Table 3. Nocturnal 6-hydroxymelatonin sulfate (6-OHMS) excretion by the quartile of temporally stable magnetic field exposure. (cr = creatinine)

\begin{tabular}{|c|c|c|c|c|c|c|c|c|}
\hline \multirow[t]{2}{*}{ Quartile of magnetic field exposure } & \multicolumn{4}{|c|}{ Nocturnal 6-OHMS/ cr concentration ${ }^{\mathrm{a}}(\mathrm{ng} / \mathrm{mg})$} & \multicolumn{4}{|c|}{ Total overnight 6-OHMS excretion ( $\mathrm{\mu g})$} \\
\hline & 1 & 2 & 3 & 4 & 1 & 2 & 3 & 4 \\
\hline Work & 29.2 & 27.5 & 30.9 & 27.2 & 17.6 & 17.3 & 16.6 & 15.1 \\
\hline Home & 32.8 & 28.4 & 30.1 & $24.5^{* *}$ & 18.4 & 15.6 & 18.6 & 14.9 \\
\hline Sleep & 29.1 & 28.3 & 29.2 & 26.3 & 17.5 & 16.8 & 16.2 & 15.7 \\
\hline
\end{tabular}

a Least-square means based on adjustment for age, month of participation, and mean light exposure during the same period.

** $P \leq 0.01$ for 1 st versus 4 th quartile.

between the highest and lowest quartiles were not statistically significant.

Additional analyses were performed to evaluate whether temporally stable magnetic field exposures over a larger portion of the day influenced 6-OHMS excretion. The subjects who were in the lowest quartile of RCMS exposure both at home and at work had mean nocturnal 6-OHMS/cr concentrations that were $39 \%$ lower than those in the highest quartile at home and at work (23.3 $\mathrm{ng} / \mathrm{mg}$ versus $38.2 \mathrm{ng} / \mathrm{mg}, \mathrm{P}=0.02$ ) (figure 1 ). Similar

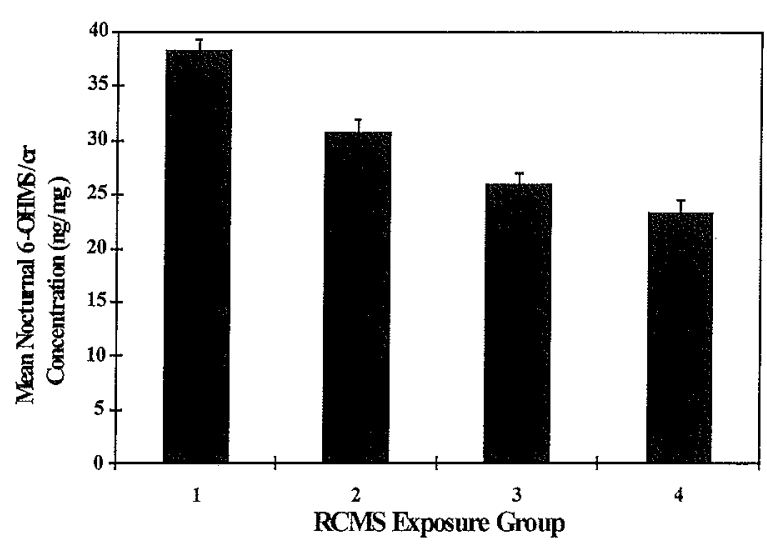

Figure 1. Least-square means of the nocturnal 6-hydroxymelatonin sulfate (6-OHMS) concentrations (ng 6-OHMS per mg creatinine) adjusted for the effects of age, month of participation, and ambient light exposure. The data have been summarized by group of magnetic field exposure using the standardized rate-of-change metric (RCMS): $4=$ lowest quartile of RCMS exposure both at work and at home; $1=$ highest quartile of RCMS exposure both at work and at home; $3=$ lowest quartile of RCMS at work or at home, but not both; and $2=$ all remaining subjects $(P=0.02$ for group 1 versus group 4$)$. results were obtained when these analyses were performed using the mean overnight 6-OHMS excretion as the dependent variable $(12.9 \mu \mathrm{g}$ versus $20.5 \mu \mathrm{g}, \mathrm{P}=0.03)$. A similar trend was noted for the subjects with temporally stable magnetic field exposure both at home and during sleep (results not shown).

\section{Discussion}

Our findings indicate that the temporal stability of magnetic fields may be important for eliciting biological effects in humans. This hypothesis was based on the findings of Litovitz et al, who measured ornithine decarboxylase (ODC) activity in vitro after exposure to magnetic fields in which the frequency was shifted at various time intervals (30). The ODC activity doubled when the frequency of a $10-\mu$ T magnetic field remained stable for intervals of at least 10 seconds (30); this finding suggests that $60-\mathrm{Hz}$ magnetic fields must remain stable over time in order elicit effects $(31,32)$.

Based in part on these findings, the RCMS was developed as an estimate of the temporal stability of exposure. Consistent with this hypothesis, low RCMS values were associated with reduced 6-OHMS excretion. Time constants calculated from the lower quartile of the RCMS at work or at home indicated that exposures remaining highly correlated for intervals of at least 3 to 5 minutes on the average (assuming a first-order autoregressive model) were associated with reduced 6-OHMS levels. When analyzed separately, magnetic field intensity, intermittence, or cumulative exposure had little or no influence on 
6-OHMS excretion although the intensities were relatively low. However, the interaction between residential magnetic field intensity and temporal stability was associated with a reduction in both 6-OHMS variables and therefore suggests that the effects of temporally stable magnetic fields are enhanced at higher field strengths.

Our results indicate that the timing of exposure to temporally stable fields may be important for suppressing 6-OHMS excretion. Light exposures that occur at times when people are expected to be at home (ie, near dawn and dusk) influence nocturnal melatonin production (3739 ), and the magnetic field suppression of melatonin may be mediated by retinal photoreceptors (40-42). If so, magnetic field exposures may need to coincide with specific periods of photosensitivity for melatonin suppression to occur. In controlled human experiments, magnetic field exposures that occurred prior to the onset of nocturnal melatonin production resulted in a delay in onset and a suppression in peak nocturnal plasma melatonin concentrations (43). Nocturnal melatonin onset usually occurs between 1600 and 2000 hours (2), which corresponds to the time of day when most of the subjects were at home. Other investigators have failed to elicit a reproducible suppression of nocturnal melatonin production in humans using only overnight magnetic field exposures that started at 2300 hours, after the nocturnal melatonin onset (44-46). Similarly, we found no statistically significant reductions in 6-OHMS in association with exposures that occurred only during sleep.

Reductions in mean nocturnal 6-OHMS levels were modest after RCMS magnetic field exposures at work. The greatest reductions in the mean 6-OHMS levels were observed when RCMS exposures at work and at home were combined (figure 1). This finding suggests that the effects of temporally stable magnetic fields are integrated over a longer time span than the approximate 8-hour periods that were used in this study and that exposures occurring during the day influence melatonin production at night. Animal experiments indicate that several weeks of exposure to $50-$ to $60-\mathrm{Hz}$ electric or magnetic fields over a large portion of the day appear to be the most effective means of suppressing melatonin (47-52) although there are some inconsistencies $(53-56)$. Short-term exposures have been ineffective $(57,58)$ unless repeated daily for several weeks (59).

Melatonin suppression has been reported for experimental animals after exposure to rapidly switched magnetic fields $(27,28)$. Our results do not support a role for intermittent exposures in suppressing 6-OHMS excretion. However, intermittent changes in magnetic fields at intervals of less than 15 seconds could not be evaluated. The rate-of-change metric was designed to capture switching events but, like the EMDEX meter, it does not specifically quantify transient exposures. Thus the negative findings for RCM in this study and the positive findings of others $(27,28)$ suggest that future studies should more carefully characterize exposure to high-frequency transients.

One strength of our study was the ability to measure light exposure and adjust for its effects on melatonin production. However, the light sensor response was matched to that of the human eye and was not maximal at all wave lengths that produce the greatest melatonin inhibition (60). Thus the effects of the measured light on 6-OHMS excretion may have been somewhat attenuated due to the misclassification of exposures.

The reductions in mean nocturnal 6-OHMS excretion associated with RCMS magnetic field exposures in this study (approximately $20-40 \%$ ) were consistent with those reported elsewhere (15-17), and the results were in general agreement whether nocturnal 6-OHMS/cr concentration or overnight 6-OHMS excretion was used as the outcome variable. Residential, rather than occupational, magnetic field exposures were most strongly associated with a reduction in nocturnal 6-OHMS excretion, which does not support the hypothesis that workplace exposures reduce 6-OHMS levels. However, the mean workplace exposures were lower than those reported by others (1821 ), and they were only marginally higher than the mean residential exposures. Thus it was not possible to determine the effects of higher workplace magnetic field exposures on 6-OHMS excretion in our population. The finding that temporally stable magnetic field exposures, as measured by RCMS, are associated with reduced 6OHMS excretion is unique and requires confirmation. Further work is also needed to determine whether 6OHMS excretion is chronically suppressed in electric utility workers and to determine whether the effects are due to a reduction in the biosynthesis of melatonin, a phase shift in nocturnal melatonin production, or an increase in melatonin metabolism. Melatonin suppression may serve as a valuable tool for understanding human biological responses to magnetic fields.

\section{Acknowledgments}

The authors gratefully acknowledge the cooperation of the participating utilities, their employees who participated in this study and their representatives: John Fooks, Platte River Power Authority; Dennis Sumner, City of Fort Collins; and Larry Graff, Poudre Valley Rural Electric Authority. The urinary 6-OHMS assays were performed under the direction of Dr Terry Nett, Director of the Radioimmunoassay Laboratory for the CSU Department of Physiology. The authors thank Ms Katherine Sutherland for her technical assistance, and Drs Lee Wilke and Martin Fettman for their assistance with the creatinine assays. Dr Gerri Lee of the California Department of Health 
provided the EMDEX meters; the Platte River Power Authority provided the light meters; Dr Scott Davis of the Fred Hutchinson Cancer Research Center provided the design for adapting the light meters to the EMDEX monitors, and Mr Pablo Lopez of the University of Washington provided assistance with the light meter adaptation. Dr Lilia Hristova of the California Department of Health provided programming assistance.

This work was supported by the US Department of Energy, Office of Energy Management, under contract 19X-SS755V with the Martin Marietta Corporation and by research grant 1 R01ES08117 from the National Institute of Environmental Health Sciences, National Institutes of Health in the United States.

\section{References}

1. Reiter RJ. Alterations of the circadian melatonin rhythm by the electromagnetic spectrum: a study in environmental toxicology. Regul Toxicol Pharmacol 1992;15:226-44.

2. Cagnacci A. Melatonin in relation to physiology in adult humans. J Pineal Res 1996;21:200-13.

3. Brezinski A. Melatonin in humans. New Eng1 J Med 1997;336:186-95.

4. Blask DE. Melatonin in oncology. In: Yu H, Reiter RJ, editors. Melatonin biosynthesis, physiological effects, and clinical applications. Boca Raton (FL): CRC Press, 1993.

5. Panzer $A$, Viljoen $M$. The validity of melatonin as an oncostatic agent. J Pineal Res 1997;22:184-202.

6. Conti A, Maestroni GJM. The clinical neuroimmunotherapeutic role of melatonin in oncology. J Pineal Res 1995;19:103-10.

7. Maestroni GJM. The immunoendocrine role of melatonin. J Pineal Res 1993;14:1-10.

8. Guerrero JM, Reiter RJ. A brief survey of pineal glandimmune system interrelationships. Endocr Res 1992;18:91113.

9. Nelson RJ, Demas GE, Klein SL, Kriegsfeld LJ. The influence of season, photoperiod, and pineal melatonin on immune function. J Pineal Res 1995;19:149-65.

10. Reiter RJ, Melchiorri D, Sewerynek E, Poeggeler B, Barlow-Walden L, Chuang $\mathrm{J}$, et al. A review of the evidence supporting melatonin's role as an antioxidant. J Pineal Res 1995;18:1-11.

11. Tan DX, Reiter RJ, Chen LD, Poeggeler B, Manchester LC, Barlow-Walden LR. Both physiological and pharmacological levels of melatonin reduce DNA adduct formation induced by the carcinogen safrole. Carcinogenesis 1994; $15: 215-8$.

12. Manev H, Tolga U, Kharlamov A, Joo J-Y. Increased brain damage after stroke or excitotoxic seizures in melatonindeficient rats. FASEB J 1996;10:1546-51.

13. Cohen M, Lippman M, Chabner B. Role of the pineal gland in the aetiology and treatment of breast cancer. Lancet 1978;2:814-6.

14. Shah PN, Mhatre MC, Kothari LS. Effect of melatonin on mammary carcinogenesis in intact and pinealectomized rats in varying photoperiods. Cancer Res 1984;44:3403-10.
15. Reiter RJ. Melatonin suppression by static and extremely low frequency electromagnetic fields: relationship to the reported increased incidence of cancer. Rev Environ Health 1994;10:171-86.

16. Wilson BW, Wright CW, Morris JE, Buschbom RL, Brown DP, Miller DL, et al. Evidence for an effect of ELF electromagnetic fields on human pineal gland function. $J$ Pineal Res 1990;9:259-69.

17. Pfluger DH, Minder CE. Effects of exposure to $16.7 \mathrm{~Hz}$ magnetic fields on urinary 6-hydroxymelatonin sulfate excretion of Swiss railway workers. J Pineal Res 1996;21:91100.

18. Deadman JE, Camus M, Armstrong BG, Heroux P, Cyr D, Plante M, et al. Occupational and residential $60 \mathrm{-Hz}$ electromagnetic fields and high frequency electric transients: exposure assessment using a dosimeter. Am Ind Hyg Assoc J 1988;49:409-19.

19. Bracken TD. Exposure assessment for power frequency electric and magnetic fields. Am Ind Hyg Assoc J 1993;54:197-204.

20. Sahl JD, Kelsh MA, Smith RW, Aseltine DA. Exposure to $60 \mathrm{~Hz}$ magnetic fields in the electric utility work environment. Bioelectromagnetics 1994;15:21-32.

21. Bowman JD, Garabrant DH, Sobel E, Peters JM. Exposures to extremely low frequency electromagnetic fields in occupations with elevated leukemia rates. Appl Ind Hyg 1988;3:189-94.

22. Savitz DA. Overview of epidemiological research on electric and magnetic fields and cancer. Am Ind Hyg Assoc J 1993;54:197-204.

23. Kheiffets LI, Abdelmonem AA, Buffler PA, Zhang ZW. Occupational electric and magnetic field exposure and brain cancer: a meta-analysis. J Occup Environ Med $1995 ; 37: 1327-41$.

24. Feychting M, Ahlbom A. Magnetic fields and cancer in children residing near Swedish high-voltage power lines. Am J Epidemiol 1993;13:467-81.

25. Floderus B, Persson T, Stenlund C, Linder G, Johansson C, Kiviranta J, et al. Occupational exposure to electromagnetic fields in relation to leukemia and brain tumors: a casecontrol study in Sweden. Cancer Causes Control $1993 ; 4: 465-76$.

26. Matanoski GM, Elliott EA, Breysse PN, Lynberg MC. Leukemia in telephone linemen. Am J Epidemiol 1993;137:609-19.

27. Lerchl A, Nonaka KO, Reiter RJ. Pineal gland "magnetosensitivity" to static magnetic fields is a consequence of induced electric currents (eddy currents). J Pineal Res 1991;10:109-16.

28. Rogers WR, Reiter RJ, Smith HD, Barlow-Walden L. Rapid-onset/offset, variably scheduled $60 \mathrm{~Hz}$ electric and magnetic field exposure reduces nocturnal serum melatonin concentration in nonhuman primates. Bioelectromagnetics 1995;3 suppl:119-22.

29. Wilson BW, Lee GM, Yost MG, Davis KC. Heimbigner T, Buschbom RL. Magnetic field characteristics of electric bed heating devices. Bioelectromagnetics 1996;17:174-9.

30. Litovitz TA, Krause D, Mullins JM. Effect of coherence of the applied magnetic field on ornithine decarboxylase activity. Biochem Biophys Res Commun 1991;178:862-5.

31. Litovitz TA, Krause D, Montrose CJ, Mullins JM. Temporally incoherent magnetic fields mitigate the response of biological systems to temporally coherent magnetic fields. Bioelectromagnetics 1994;15:399-409. 
32. Litovitz TA, Penafiel M, Krause D, Zhang D, Mullins JM. The role of temporal sensing in bioelectromagnetic effects. Bioelectromagnetics 1997; 18:388-95.

33. Arendt J, Bojkowski C, Franey C, Wright J, Marks V. Immunoassay of 6-hydroxymelatonin sulfate in human plasma and urine: abolition of the urinary 24-hour rhythm with atenolol. J Clin Endocrinol Metab 1985;60:1166-73.

34. Aldous ME, Arendt J. Radioimmunoassay for 6-sulphatoxymelatonin in urine using an iodinated tracer. Ann Clin Biochem 1988;25:298-303.

35. Bojkowski CJ, Arendt JA, Shih MC, Markey SP. Melatonin secretion in humans assessed by measuring its metabolite, 6sulfatoxymelatonin. Clin Chem 1987;33:1343-8.

36. Fellenberg AJ, Phillipou G, Seamark RF. Specific quantitation of urinary 6-hydroxymelatonin sulfate by gas chromatography mass spectrometry. Biomed Mass Spectrom 1980;7:84-7.

37. Wehr TA, Moul DE, Barbato G, Giesen HA, Seidel JA, Barker $\mathrm{C}$, et al. Conservation of photoperiod-responsive mechanisms in humans. Am J Physiol 1993;265:R846-57.

38. Laakso M-L, Porkka-Heiskanen T, Alila A, Stenberg D, Johansson G. Twenty-four-hour thythms in relation to the natural photoperiod: a field study in humans. J Biol Rhythms $1994 ; 9: 283-93$

39. Kollar M, Marma M, Laitinen JT, Kundi M, Piegler B, Haider M. Different patterns of light exposure in relation to melatonin and cortisol rhythms and sleep of night workers. J Pineal Res 1994:16:127-35.

40. Olcese J, Reuss S, Vollrath L. Evidence for the involvement of the visual system in mediating magnetic field effects on pineal melatonin synthesis in the rat. Brain Res 1985;333:382-84.

41. Reuss S, Olcese J. Magnetic field effects on rat pineal gland: role of retinal activation by light. Neurosci Lett 1986;64:97-101.

42. Phillips JB, Deutschlander ME. Magnetoreception in terrestrial vertebrates: implications for possible mechanisms of EMF interaction with biological systems. In: Stevens R, Wilson BW, Anderson LE, editors. The melatonin hypothesis and breast cancer. Columbus (OH): Batelle Press, 1997.

43. Wood AW, Armstrong SM, Sait ML, Devine L, Martin MJ. Changes in human plasma melatonin profiles in response to $50 \mathrm{~Hz}$ magnetic field exposure. J Pineal Res. In press.

44. Graham C, Cook MR, Riffle DW, Gerkovich MM, Cohen HD. Nocturnal melatonin levels in human volunteers exposed to intermittent $60 \mathrm{~Hz}$ magnetic fields. Bioelectromagnetics 1996;17:263-73.

45. Graham C, Cook MR, Riffle DW. Human melatonin during continuous magnetic field exposure. Bioelectromagnetics 1996; 18:166-71.

46. Selmaoui B, Touitou Y. Magnetic fields and pineal function in humans: evaluation of nocturnal acute exposure to extremely low frequency magnetic fields on serum melatonin and urinary 6-sulfatoxymelatonin circadian rhythms. Life Sci $1996 ; 58: 1539-49$.

47. Selmaoui B, Touitou Y. Sinusoidal $50 \mathrm{~Hz}$ magnetic fields depress rat pineal NAT activity and serum melatonin: role of duration and intensity of exposure. Life Sci 1995;57:1351-58.

48. Kato M, Honma K, Shigemitsu T, Shiga Y. Effects of circularly polarized $50-\mathrm{Hz}$ magnetic field on plasma and pineal melatonin levels in rats. Bioelectromagnetics 1993:14:97-106.

49. Kato M, Honma K, Shigemitsu T, Shiga Y. Circularly polarized $50-\mathrm{Hz}$ magnetic field exposure reduces pineal gland and blood melatonin concentrations in Long-Evans rats. Neurosci Lett 1994;166:59-62.

50. Loescher W, Wahnschaffe U, Mevissen M, Lerchl A, Stamm A. Effects of weak alternating magnetic fields on nocturnal melatonin production and mammary carcinogenesis in rats. Oncology 1994;51:288-95.

51. Mevissen M, Lerchl A, Loescher W. Study of pineal function and DMBA-induced breast cancer formation in rats during exposure to a $100-\mathrm{mG}, 50 \mathrm{~Hz}$ magnetic field. $\mathrm{J}$ Toxicol Environ Health 1996;48:169-85.

52. Wilson BW, Chess EK, Anderson LE. $60-\mathrm{Hz}$ electric field effects on pineal melatonin rhythms: time course for onset and recovery. Bioelectromagnetics 1986;7:239-42.

53. Lee JM, Stormshak F, Thompson JM, Thinesen P, Painter LJ, Olenchek EG, Hess DL, Forbes R, Foster DL. Melatonin secretion and puberty in female lambs exposed to environmental electric and magnetic fields. Biol Reprod $1993 ; 49: 857-64$.

54. Lee JM, Stormshak F, Thompson JM, Hess DL, Foster DL. Melatonin and puberty in female lambs exposed to EMF: a replicate study. Bioelectromagnetics 1995;16:119—23.

55. Rogers WR, Reiter RJ, Smith HD, Barlow-Walden L. Regularly scheduled, day-time, slow-onset $60 \mathrm{~Hz}$ electric and magnetic field exposure does not depress serum melatonin concentrations in nonhuman primates. Bioelectromagnetics 1995;3 suppl:111-8.

56. Grota LJ, Reiter RJ, Keng P, Michaelson S. Electric field exposure alters serum melatonin but not pineal melatonin synthesis in male rats. Bioelectromagnetics 1994;15:42737.

57. Bakos J, Nagy N, Thuroczy G, Szabo LD. Sinusoidal $50 \mathrm{~Hz}$, $500 \mu \mathrm{T}$ magnetic field has no acute effect on urinary 6sulfatoxymelatonin in Wistar rats. Bioelectromagnetics $1995 ; 16: 377-80$

58. Truong H, Yellon SM. Effect of various acute $60 \mathrm{~Hz}$ magnetic field exposures on the nocturnal melatonin rise in the adult Djungarian hamster. J Pineal Res 1997;22:17783.

59. Martinez Soriano F, Gimenez Gonzalez M, Armanazas E, Ruiz Torner A. Pineal 'synaptic ribbons' and serum melatonin levels in the rat following the pulse action of 52-Gs $(50 \mathrm{~Hz})$ magnetic fields: an evolutive analysis over 21 days. Acta Anatomica 1992;143:289-93.

60. Brainard GC, Lewy AJ, Menaker M, Frederickson RA, Miller LS, Weleber RG, et al. Effect of light wavelength on the suppression of nocturnal plasma melatonin in normal volunteers. Ann NY Acad Sci 1985;453:376-8.

Received for publication: 27 June 1997 